\title{
AN OPTIMAL RPC BASED APPROACH TO INCREASE FAULT TOLERANCE IN WirELESS AD-HOC NETWORK
}

\author{
Mr. Rabindra Kumar shial ${ }^{1}$, Mr. K Hemant Ku.Reddy ${ }^{2}$, Mr. K.L.Narayana ${ }^{3}$ \\ Dept. of Computer Science \& Engineering \\ National Institute of Science \& Technology, Odisha.
}

rkshial@yahoo.com, khemant.reddy@gmail.com, lakshmi2912@hotmail.com

\begin{abstract}
In wireless network, fault tolerant topology control is an important and a challenging task. The wireless nodes and links could experience frequent failures since Wireless networks are usually deployed under extreme environments. Therefore, fault tolerance must be considered for many applications. In wireless network Topology control has been proved effective in saving node power. The main idea of topology control is that instead of using its maximal transmission power, each node sets its power to a certain level such that the global topology satisfies a certain constraint. To increase fault tolerance, nodes in the network will consume more power. Here we need to give the optimum distance between the nodes of the wireless network in order to reduce the consumption of the power of each node.
\end{abstract}

This paper deals with the approach for the finding the minimum distance between the nodes of the wireless network by the use of mat lab coding through the graph theory considering the minimum weight based algorithm.

\section{KEYWORDS}

Ad-hoc wireless network, RPC, graph theory, spinning tree, fault tolerance, PSO, WMA.

\section{INTRODUCTION}

Basically in wireless networks $[8,15]$, the communication models can be categorized into four classes namely all-to-all communication network, which represents end-to-end communication of every pair of nodes in the network. Secondly one-to-one in which the communication from a given source node to a given destination node can be done. The other way of wireless communication model all-to-one, which indicates the communication from all nodes to a given (root) node. And in one-to-all, which indicates the communication from the root to all the other nodes.

The rest of the paper is organized as follows: section 2 describes power assignment to nodes in a typical power system and its network topology. Section 3 describes an approach to minimize the weight and find the minimum distance between nodes in a network. Section 4 describes power optimization using Particle swarm optimization. Section 5 summarizes the simulation result. Section 6 describes the conclusion and future developments.

DOI : $10.5121 /$ ijans.2012.2201 


\section{Power Assignment to Node:}

Considering a set $V=\left\{v_{1}, v_{2}, v_{3}, \ldots, v_{n}\right\}$ of $n$ wireless nodes distributed in a 2D plane. Let $w_{u v}$ is the power [10] needed to support the communication between two nodes $u$ and $v$ is a monotone increasing function of the Euclidean distance $\|u v\|$. In other words, $w_{u v}>w_{x y}$ if $\|u v\|$ $>\|x y\|$ and $w_{u v}=w_{x y}$ if $\|u v\|=\|x y\| . w_{u v}=c+\|u v\|^{\beta}$, where $c$ is a positive constant real number, and real number $\beta €\{2 ; 5\}$ depends on the transmission environment, and $\|u v\|$ is the Euclidean distance between points $u$ and $v$. Assuming all nodes have omni-directional antennas, i.e., if the signal transmitted by a node $u$ can be received by a node $v$, then it will be received by all nodes $x$ with $\|u x\| \leq\|u v\|$. In addition, we assumed that all nodes can adjust the transmission power dynamically. The present work represents the minimum distance and the minimum distance path of one to all communication network model.

\subsection{Network Topology:}

The network considered in this case is a wireless network consisting of $\mathrm{N}$ nodes each is equipped with an omni-directional antenna with a maximal transmission range of $r_{\max }$. The power required for a node to attain a transmission range of ' $\mathrm{r}$ ' is at least $\mathrm{Cr}^{\alpha}$, where $\mathrm{C}$ is a constant, $\alpha$ is the power attenuation exponent and usually chosen between 2 and 4 . For any two nodes $u$ and $v$, there exists a link from $u$ to $v$ if the distance $d(u, v) \leq r_{u}$, where $r_{u}$ is the transmission range for node $u$, determined by its power level. If the links are asymmetric, the existence of a link from $u$ to $v$ does not guarantee the existence of a link from $\mathrm{v}$ to $\mathrm{u}$. The network is assumed to have symmetric links and is static, i.e., the nodes in the network are stationary. Given the coordination of the nodes in the plane and the transmission power of the nodes, the network can be mapped into a cost graph $\mathrm{G}=(\mathrm{V}, \mathrm{E}, \mathrm{c})$, where $\mathrm{V}$ denotes the set of wireless nodes, $\mathrm{E}$ denotes the set of wireless links induced by the transmission power, and the weight $\mathrm{c}$ for a given edge $(\mathrm{u}, \mathrm{v})$ is computed as $\mathrm{C}_{\mathrm{d}}(\mathrm{u}$, $\mathrm{v})^{\alpha}$, where $\mathrm{d}$ is the distance. By this mapping, a symmetric wireless network is represented by an undirected graph.

Wireless Network [7] have an important feature called Wireless Multicast Advantage (WMA) because of its broadcast media. WMA is often utilized to save power. For a node to send data to multiple nodes (one to all) in its transmission range, instead of sending data multiple times, it only needs to send it once and all nodes in its transmission range can receive the same data. In light of WMA, the power and weight are different in wireless networks, where weight is link based, while power is node based.

\subsection{Graph Theory}

A simple graph $G$ is a pair $G=(V, E)$ where ' $V$ ' is a finite set, called the vertices of $G$, ' $E$ ' is a subset of $\mathrm{P}_{2}(\mathrm{~V})$ (i.e., a set $\mathrm{E}$ of two-element subsets of $\mathrm{V}$ ), called the edges of $\mathrm{G}$ [4], where the notation $P_{k}(V)$ stands for the set of all k-element subsets of the set $V$. A graph is triple $G=(V, E$, $\varphi)$ where ' $V$ ' is a finite set, called the vertices of $G$, ' $E$ ' is a finite set, called the edges of $G$, and ' $\varphi$ ' is a function with domain $\mathrm{E}$ and co domain $\mathrm{P}_{2}(\mathrm{~V})$. Given the coordination of the nodes in the plane and the transmission power of the nodes, the network can be mapped into a cost graph $\mathrm{G}=$ $(\mathrm{V}, \mathrm{E}, \mathrm{c})$, where $\mathrm{V}$ denotes the set of wireless nodes, $\mathrm{E}$ denotes the set of wireless links induced by the transmission power, and the weight $\mathrm{c}$ for a given edge $(\mathrm{u}, \mathrm{v})$ is computed as $\mathrm{C}_{\mathrm{d}}(\mathrm{u}, \mathrm{v})^{\alpha}$, where $\mathrm{d}$ is the distance. By this mapping, a symmetric wireless network is represented by an undirected graph. 


\section{Minimum Weight Based Approach:}

The main idea of Minimum Weight Based Algorithm (MWBA) is to construct a k-out connected [1] sub-graph with the goal to minimize its weight, and then analyze its performance for minimum power k-out connectivity problem. Given $G=(\mathrm{V}, \mathrm{E}), \mathrm{k}$, and root node $\mathrm{r} € \mathrm{~V}$, MW utilizes FT which constructs a minimum weight directed k-out connected sub-graph for a directed graph as follows:

1.Construct $\mathrm{G}^{\prime}$ by replacing each edge in $\mathrm{G}$ with two opposite directed edges. The weight of each directed edge is the same weight as the original edge.

2.DFT $=$ FT $\left(\mathrm{G}^{\prime}, \mathrm{k}, \mathrm{r}\right)$, DFT is the directed k-out connected sub-graph with optimal weight

3. Construct the undirected version of DFT, called GFT. An undirected graph is constructed from a directed graph as follows: if there is a directed edge $u v$ in DFT, then there exists an undirected edge $u v$ in GFT. It is obvious that GFT is an undirected k-out connected sub-graph.

Example: Let us consider a network graph as shown in figure 1

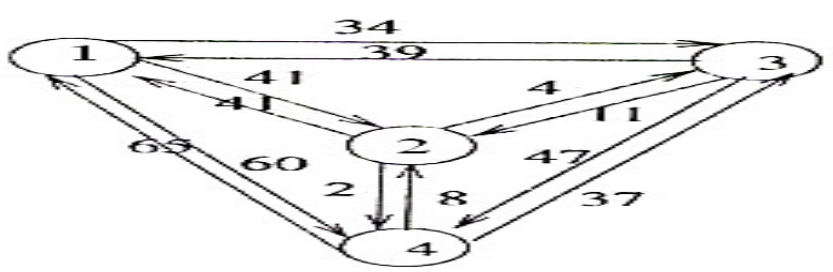

[Figure 1: Network Graph with node weights.]

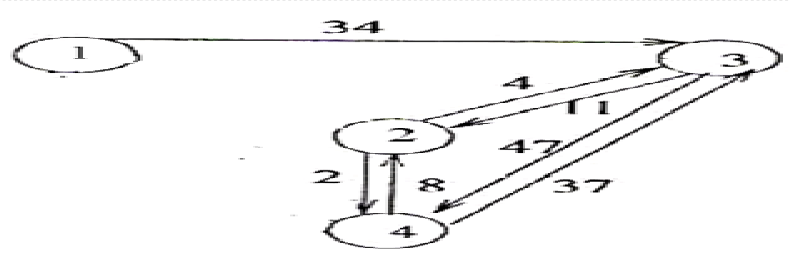

[Figure 2: Sub-Graph after $1^{\text {st }}$ Iteration. ]

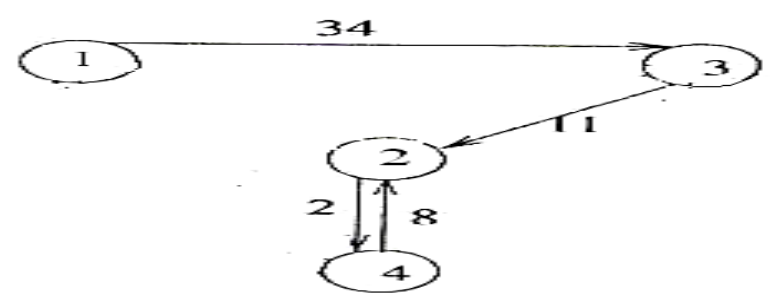

[Figure 3: Sub-Graph after $2^{\text {nd }}$ Iteration] 


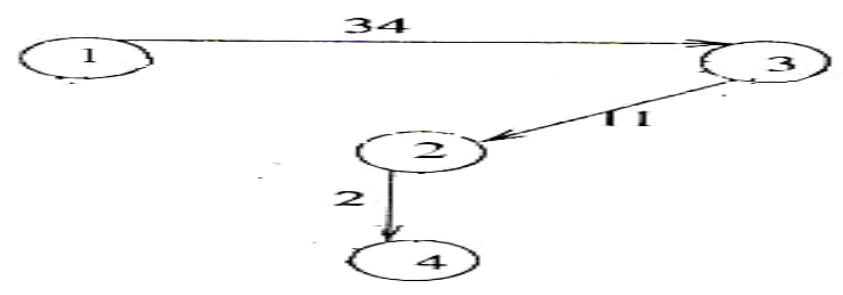

[Figure 4: Sub-Graph after $3^{\text {rd }}$ Iteration.]

As explained in the graph theory the following figures 2, 3 and 4 are the direct k-out connected sub-graphs [2,3] which are represented as iterations.

After $3^{\text {rd }}$ iteration: Since all nodes are traversed after the third iteration in the figure- 4 which is the required optimum network.

\subsection{Approach: Optimum Distance}

In this section we considered six node wireless networks as shown in figure- 5 for complexity and of course it can be more than six which we considered as ' $N$ ' node for the simulation results.

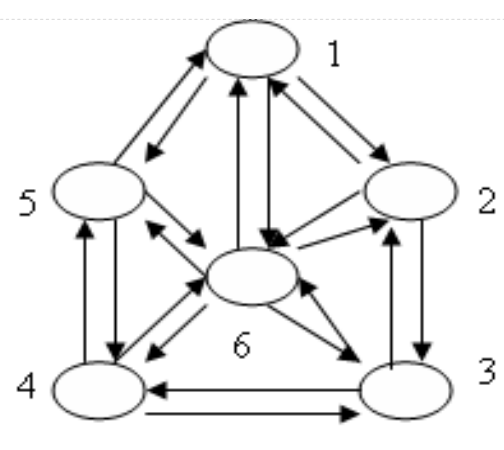

[Figure 5: Wireless Network.]

\begin{tabular}{|c||c|c|c|c|c|c|}
\hline Nodes & 1 & 2 & 3 & 4 & 5 & 6 \\
\hline 1 & 0 & 10 & 12 & 5 & 2 & 6 \\
\hline 2 & 8 & 0 & 11 & 10 & 16 & 20 \\
\hline 3 & 18 & 26 & 0 & 3 & 5 & 31 \\
\hline 4 & 14 & 15 & 7 & 0 & 9 & 1 \\
\hline 5 & 23 & 24 & 4 & 6 & 0 & 17 \\
\hline 6 & 19 & 30 & 14 & 15 & 36 & 0 \\
\hline
\end{tabular}

[Table 1: Power vs. No. of Nodes.]

The distances between the nodes 2 and 1 is 8 as shown by the arrows in the table-1, which are the inputs for the logic based on the minimum weight based approach for which the algorithm has been used.

\subsection{Algorithm: Minimum Distance Calculation}

START:

1. Set $\mathrm{N}:=$ Input the number of nodes.

2. Find D:= Distance between all the nodes $\& D_{i}$ : is the distance of node $N_{i}$

3. Find MAX:=Maximum distances among all nodes.

$$
\operatorname{MAX}=\max \left\{d\left(N_{1}\right), d\left(N_{2}\right), d\left(N_{3}\right), \ldots . ., d\left(N_{n}\right)\right\}
$$


4. Set $\operatorname{Max}=\operatorname{Max}+1$;

5. Replace the distance of unconnected nodes with Max.

6. Level : L1

7. Min:= Minimum distance of connected node with node 1 .

$$
\mathrm{MIN}=\min _{i}\left\{d\left(N_{i}\right)\right\}, i=1,2, \ldots, n
$$

8. Display the node

9. goto node having minimum distance

10. If( all nodes are visited )

$$
\operatorname{exit}(0) \text {; }
$$

11. else

$$
\text { goto L1; }
$$

12. STOP.

\section{Optimizing Power:}

A wireless network consists of $\mathrm{N}$ nodes, each of which is equipped with an omni-directional antenna with a maximal transmission range of $r_{\max }$. The power [11] required for a node to attain a transmission range of $\mathrm{r}$ is at least $\mathrm{Cr}^{\alpha}$, where $\mathrm{C}$ is a constant, $\alpha$ is the power attenuation exponent and usually chosen between 2 and 4 . For any two nodes $u$ and $v$, there exists a link from $u$ to $v$ if the distance $d(u, v) \leq r_{u}$, where $r_{u}$ is the transmission range for node $u$, determined by its power level. If the links are asymmetric, the existence of a link from $\mathrm{u}$ to $\mathrm{v}$ does not guarantee the existence of a link from $v$ to $u$. In this paper, we consider symmetric links and assume the wireless network is static, i.e., the nodes in the network are stationary. Given the coordination of the nodes in the plane and the transmission power of the nodes, the network can be mapped into a cost graph $\mathrm{G}=(\mathrm{V}, \mathrm{E}, \mathrm{c})$, where $\mathrm{V}$ denotes the set of wireless nodes, $\mathrm{E}$ denotes the set of wireless links induced by the transmission power, and the weight $\mathrm{c}$ for a given edge $(\mathrm{u}, \mathrm{v})$ is computed as $\mathrm{Cd}(\mathrm{u}, \mathrm{v})^{\alpha}$, where $\mathrm{d}$ is the distance. By this mapping, a symmetric wireless network is represented by an undirected graph.

\subsection{Particle Swarm Optimization (PSO):}

PSO is a method for doing numerical optimization without explicit knowledge of the gradient of the problem to be optimized. PSO is due to Kennedy, Eberhart and Shi was originally intended for simulating social behaviour, but the algorithm was simplified and it was realized that the particles were actually performing optimization. The book by Kennedy and Eberhart describes many philosophical aspects of PSO and swarm intelligence. PSO optimizes a problem by maintaining a population of candidate solutions called particles and moving these particles around in the search-space according to simple formulae. The movements of the particles are guided by the best found positions in the search-space, which are continually being updated as better positions are found by the particles.

Explanation: Evolutionary computation exploit a set of potential solutions and detect the optimal ones through cooperation and competition among the individuals of the population. Particle swarm optimization (PSO) is one of the population based stochastic optimization technique inspired by social behavior of "bird flocking". PSO shares many similarities with evolutionary 
computation techniques such as Genetic Algorithm(GA). A population of random individuals is initially generated and these individuals probe the search space during their evolution to identify the optimal solution. Compared to GA, PSO does not employ evolution operators such as cross over and mutation and does not need information about the objective function gradient. Other advantages characterized by PSO such as the easy implementation and the low requirement of computational resources. In PSO, the individuals, called the particles, are collected into a swarm and fly through the problems by following the optima particles. Each individual has a memory, remembering the best position of the search space it has ever visited. In particular, particle remembers the best position among those it has visited, refer to as a pbest, and the best position by its neighbors. There are 2 versions for keeping the neighbors best position, namely lbest and gbest. The first(lbest) is related to the best position of the particle in the neighbors of the particle itself while gbest refers to the best position recorded by the entire swarm. Each individual of the population has an adaptable velocity (position change), according to which it moves in the search space. Thus, its movement is an aggregated acceleration towards its best previously visited position and towards the best individual of a topological neighborhood. Compared to GA, the information sharing mechanism of PSO is notably different. Chromosomes share information and the entire population evolves towards an optimal area in compact manner. The evolution of particles, guided only by the best solution, tends to be regulated by behavior of the neighbors. In the simplest form, the position $\mathrm{S}$ and velocity $\mathrm{V}$ of each particle are represented by the following equations by considering lbest rather than gbest as the best position of the particle referred to the neighbours.

\subsection{Basic PSO Equations}

The particle velocity is expressed as

$\mathrm{V}(\mathrm{t})=\mathrm{W} * \mathrm{~V}(\mathrm{t}-1)+\mathrm{C}_{1} * \mathrm{r}_{1}{ }^{(\text {lbest- } \mathrm{S}(\mathrm{t}-1))}+\mathrm{C}_{2} * \mathrm{r}_{2}{ }^{(\text {gbest- } \mathrm{S}(\mathrm{t}-1))}$

And the particle displacement is expressed as

$\mathrm{S}(\mathrm{t})=\mathrm{S}(\mathrm{t}-1)+\mathrm{V}(\mathrm{t})$, Where $\mathrm{V}=$ particle velocity

$\mathrm{S}=$ particle displacement

Lbest $=$ local best

Gbest $=$ global best

$\mathrm{W}=$ inertial weight

$\mathrm{C}_{1}$ and $\mathrm{C}_{2}$ are acceleration constants $=2.0$

$\mathrm{r}_{1}$ and $\mathrm{r}_{2}$ are random variables $\{0,1\}$

$\mathrm{t}=$ current iteration

\subsection{Application of PSO in Power Calculations:}

The power is expressed as:

$$
P(i)=w^{*} P(t-1)+C_{1} * r_{1}^{\left(L_{\text {best }}-S(t-1)\right)}+C 2 * r_{2}^{\left(G_{\text {best }}-S(t-1)\right)}
$$

The distance is expressed as:

$$
S(t)=S(t-1)+P(t) \text {, Where } \mathrm{P} \text { is power, } \mathrm{S} \text { is distance. }
$$


$L_{\text {best }}=$ Local best, $G_{\text {best }}=$ Global best

$\mathrm{W}=$ Inertial weight and $C_{1}, C_{2}$ are acceleration constants $=2.0$

$\mathrm{r}_{1}, \mathrm{r}_{2}$ are random variables $\& \mathrm{i}=$ current iteration

$L_{\text {best }}:$ Optimum power obtained locally \& $G_{\text {best }}$ :Optimum power obtained globally

The values taken for the power: $\mathrm{W}=\mathrm{unifrnd}(0.8,1.1), \quad \mathrm{r} 1=\operatorname{unifrnd}(0.26,0.3)$

r2=1-r1 \& $L_{\text {best }}=10, G_{\text {best }}=12 \& C_{1}=C_{2}=2$.

When any signal is transmitted from one to another the signal gets attenuated due to various atmospheric factors like temperature, pressure, rain, wind etc. Hence attenuation factor should be taken into account while establishing a network. Since the signal gets attenuated the signal needs to be amplified at every node of the network. But the signal cannot be amplified if the signal gets attenuated below a certain level. Therefore amplification factor should also be considered.

Amp=unifrnd(1,1.3), Attn=unifrnd(0.35,0.5)

Since the attenuation and amplification vary from place to place and time to time so it is appreciable to use random functions to obtain their value.

\section{Results Discussion:}

Let us consider any network with 4 nodes: The distance matrix of the given network is given by:

\begin{tabular}{|c|c|c|c|c|}
\hline Nodes & $\mathbf{1}$ & $\mathbf{2}$ & $\mathbf{3}$ & $\mathbf{4}$ \\
\hline $\mathbf{1}$ & 0 & 21 & 27 & 32 \\
\hline $\mathbf{2}$ & 40 & 0 & 38 & 12 \\
\hline $\mathbf{3}$ & 16 & 20 & 0 & 18 \\
\hline $\mathbf{4}$ & 39 & 27 & 10 & 0 \\
\hline
\end{tabular}

[Table 2: Node distance matrix.]

Using the minimum weight based approach we get the optimum path as $1 \rightarrow 2 \rightarrow 4 \rightarrow 3$. The power for the above path can be obtained using PSO. The power so obtained will be the optimum power for the network.

\begin{tabular}{|c|l|}
\hline Nodes & Power \\
\hline $\mathbf{1}$ & 0.1 \\
\hline $\mathbf{2}$ & 0.06819 \\
\hline $\mathbf{3}$ & 0.057233 \\
\hline $\mathbf{4}$ & 0.055203 \\
\hline
\end{tabular}

[Table 3:Node optimum power list. ]

The number of paths traversing all the nodes are $=3 !=6$, The paths are shown below: 
International Journal on AdHoc Networking Systems (IJANS) Vol. 2, No. 2, April 2012

\begin{tabular}{|c|c|c|c|c|}
\hline \multicolumn{2}{|c|}{ Slno/paths } & & & \\
\hline 1 & 1 & 4 & 3 & 2 \\
\hline 2 & 1 & 4 & 2 & 3 \\
\hline 3 & 1 & 3 & 4 & 2 \\
\hline 4 & 1 & 3 & 2 & 4 \\
\hline 5 & 1 & 2 & 3 & 4 \\
\hline 6 & 1 & 2 & 4 & 3 \\
\hline
\end{tabular}

[Table 4: Node traversal path list.]

The power at all nodes along different path are given below:

\begin{tabular}{|c|c|c|c|c|}
\hline Path/nodes & 1 & 2 & 3 & 4 \\
\hline 1 & 0.1 & 0.087509 & 0.055662 & 0.0659130 \\
\hline 2 & 0.1 & 0.087509 & 0.077178 & 0.092966 \\
\hline 3 & 0.1 & 0.077533 & 0.063871 & 0.076513 \\
\hline 4 & 0.1 & 0.077533 & 0.066371 & 0.057204 \\
\hline 5 & 0.1 & 0.06819 & 0.10187 & 0.064347 \\
\hline 6 & 0.1 & 0.06819 & 0.057233 & 0.055203 \\
\hline
\end{tabular}

[Table 5. Node power with different path ]

From the above table we can conclude that the power is maximum in case of path 2 . The power variation with respect to the distance for path 2 is shown in the below figure

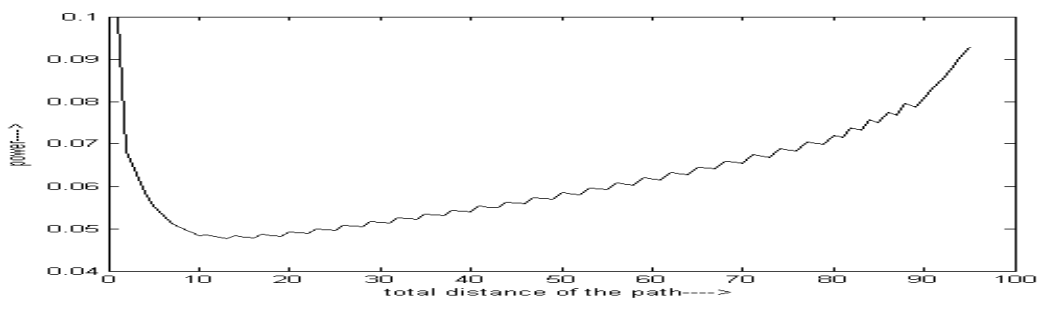

[Figure6: Power vs total distance of the path]

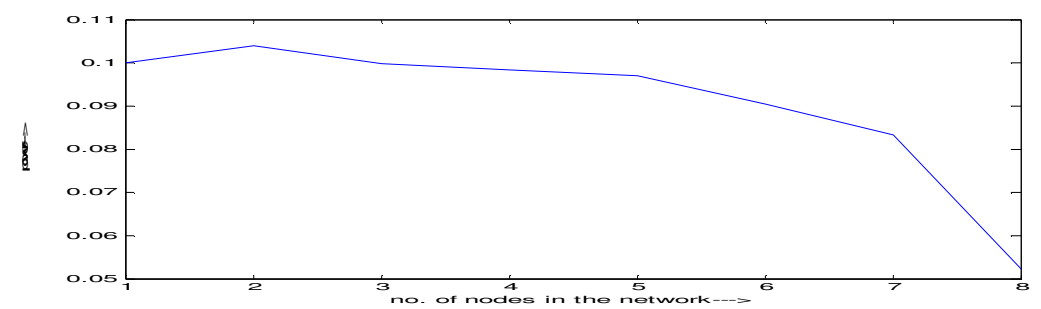

[Figure.7. Power vs. No. of Nodes in network] 
Therefore we can infer from the above plot that as the number of nodes in a network increases the power at the end node of each network decreases [13]. From the plot we can observe that if the number of nodes in a network is more than 8 then the power at the end node of the network drops off below the threshold value for which it cannot be amplified. So it is desirable to take into consideration the number of nodes in a network while establishing the network physically

\section{Conclusions:}

In this paper, we propose the use of Particle Swarm Optimization technique to minimize the distance and consumption of power in wireless ad-hoc network environment by increasing the fault tolerance of any network. The network achieved by this way is an optimized network covering optimized area with minimum power consumption. Our proposed approach starts by taking nodes in a network to be used as a network group. We also explored the results of the performance evaluation of four extensions to the standard Particle Swarm Optimization algorithm in order to reduce the energy consumption in Wireless Ad-hoc Networks. Communication distance is an important factor to be reduced in ad-hoc networks. We have simulated in mat-lab and simulation result shows an exciting result. The result of the proposed technique is also compared with two other well-known clustering algorithms for power consumption $[11,13]$ though the framework of our work different then the existing two technique. The results exhibit the promising capability of the proposed technique and clearly show that it works better than the other two clustering techniques.

\section{References:}

[1]. Feng Wang, My T.Thai, yingshu Li, Xiuzhn Cheng, Ding Zhu Du "Fault tolerant topology control for all to one and one to all communication in wireless network"

[2]. Christian Lavaul, Mario Valencia-Pabon, "A distributed approximation algorithm for the minimum degree minimum weight spanning trees"

[3]. Harvey J. Greenberg, "Greedy Algorithms for Minimum Spanning Tree"

[4]. Tero Harju, "GRAPH THEORY"

[5]. Fault-Tolerant Topology Control for All-to-One and One-to-All Communication in Wireless Networks, IEEE Transactions on mobile computing, Vol 7, No 3

[6]. A Survey of Dependability Issues in Mobile Wireless Networks Claudio Basile, Marc-Olivier Killijian, David Powell cbasile@uiuc.edu, $\square$ marco.killijian, david.powell_@laas.fr February 21, 2003

[7]. I. Akyildiz, W. Su, Y. Sankarasubramaniam, and E. Cayirci, "Wireless sensor networks: A survey," Computer Networks Journal, vol. 38, no. 4, pp. 393-422, 2002.

[8]. W. Su, S.-J. Lee, and M. Gerla, "Mobility prediction and routing in ad hoc wireless networks," Int'1 Journal of Network Management, 2000.

[9]. S. Singh, M. Woo, and C. S. Raghavendra, Power aware routing in mobile ad hoc networks, Proc. ACM Mobicom, pp. 181-190 (1998).

[10]. J. P. Monks, V. Bhargavan, and W. M. Hwu, A power controlled multiple access protocol for wireless packet networks, Proc. IEEE INFOCOM, pp. 219-228 (2001).

[11]. Weighted Clustering using Comprehensive Learning Particle Swarm Optimization for Mobile Ad Hoc Networks. Waseem Shahzad, Farrukh Aslam Khan, and Abdul Basit Siddiqui

[12]. The Implementation Of Optimization Algorithm For Energy Efficient Dynamic Ad Hoc Wireless Sensor Networks Mohaned Al. Obaidy, and Aladdin Ayesh

[13]. S. Bandyopadhyay and E. J. Coyle, 'An energy efficient hierarchical clustering algorithm for wireless sensor networks', Proceedings of the IEEE Conference on Computer Communications (INFOCOM 2003).

[14]. Lucille Verbaere Armin Wellig C. Laurent, Didier Helal and Julien Zory, 'Wireless sensor networks devices: Overview, issues, state of the art and promising technologies', ST Journal of Research, 4, No.1, (June8, 2007). 